\title{
ACTITUDES HACIA LA CALIDAD DE VIDA LABORAL EN TRABAJADORES CONTROL DE VÍA DE TRANSPORTE URBANO
}

\section{ATTITUDES TOWARD THE QUALITY OF WORKING LIFE IN URBAN TRANSPORT ROUTE- CONTROL WORKERS}

\section{ATITUDES EM RELAÇÃO A QUALIDADE DE TEMPO DE TRABALHO EM FUNCIONARIOS DE CONTROLE DE TRANSPORTE URBANO}

Por: CHAVES LOPEZ _ Lizeth Daniela, GRIJALBA ESPINOSA_ María Alejandra, BÁRCENAS ENRÍQUEZ _ David Román, MATABANCHOY TULCÁN_Sonia Maritza, ZAMBRANO GUERRERO _ Christian Alexander

Psicóloga practicante del Programa de Psicología de la Universidad de Nariño, Unión Temporal Ciudad Sorpresa, Pasto. Email: danniela.cl08@gmail.com, Colombia.

Psicóloga practicante del Programa de Psicología de la Universidad de Nariño, Unión Temporal Ciudad Sorpresa, Pasto. Email: alejandragres@hotmail.com, Colombia.

Psicólogo practicante del Programa de Psicología de la Universidad de Nariño, Unión Temporal Ciudad Sorpresa, Pasto. Email: doomsdavid@gmail.com, Colombia.

Doctoranda en Psicología - Universidad Católica de Argentina. Especialista Alta Gerencia. Magíster Gerencia del Talento Humano. Psicóloga docente tiempo completo Programa Psicología - Universidad de Nariño. Integrante del grupo de investigación CONEPSI del Programa de Psicología -Universidad de Nariño. Consultor de Talento Humano, Pasto. Email: somapsicologa@yahoo.es, Colombia.

Magister en Educación desde la diversidad - Universidad de Manizales. Psicólogo docente del Programa de Psicología de - Universidad de Nariño. Integrante del grupo de investigación Psicología y Salud e integrante del grupo de investigación CONEPSI del Programa de Psicología - Universidad de Nariño, Pasto. Email: christianzambris@hotmail.com, Colombia.

DOI: http://dx.doi.org/10.22267/rtend.171802.77

\section{RESUMEN}

El estudio tuvo como objetivo identificar las actitudes hacia la calidad de vida laboral en 94 trabajadores que desempeñan el cargo de control de vía en la Unión Temporal Ciudad Sorpresa de la ciudad de Pasto (Colombia). El estudio fue de carácter cuantitativo de tipo descriptivo transversal y la información se recolectó a través de 
la aplicación de una escala tipo Likert, comprendiendo las categorías: salud laboral, bienestar laboral y percepción del trabajo. Se encontró en la población una actitud favorable hacia la mayoría de las categorías trabajadas, sin embargo, se reportaron cifras que requieren atención como el autorreporte de morbilidad sentida y la categoría de salud laboral, específicamente identificando una actitud desfavorable en la subcategoría, condiciones de trabajo.

Palabras clave: actitudes, calidad de vida laboral, salud laboral, bienestar laboral, percepción del trabajo, sistema de transporte, control de vía.

JEL: I31, R41, R42.

\section{ABSTRACT}

This study aimed to identify attitudes towards the quality of the working life of 94 employees who serve in the position of route-control workers for the "Unión Temporal Ciudad Sorpresa" (Surprise City Temporary Union), in Pasto (Colombia). The study was quantitative, descriptive, and transversal, and information was collected through the application of a Likert scale. This scale included the following categories: occupational health, workplace wellness, and work perception. A favorable attitude toward most of the cited categories was found among the population; however, some reported figures require attention, such as self-reported perceived morbidity, and the occupational health category. An unfavorable attitude in the subcategory of working conditions was specifically identified.

Keywords: attitudes, quality of the working life, occupational health, workplace wellness, work perception, transport system, route-control worker.

JEL: I31, R41, R42.

\section{RESUMO}

0 estudo teve por objetivo identificar as atitudes em relação à qualidade de vida no trabalho com 94 funcionários, que realizam o cargo de controle rodoviário Temporária da Cidade Surpresa de Pasto (Colômbia). 0 estudo foi quantitativo de tipo descritivo transversal e as informações foram coletadas através da aplicação de uma escala de Likert, categorias, saúde ocupacional, bem-estar no local de trabalho e a percepção de entendimento trabalho. Foi encontrado na população uma atitude favorável na maioria das categorias trabalhadas, no entanto, os números que requerem atenção como a morbidez auto-relatados e categoria de saúde no trabalho, foram relatados, identificando especificamente uma atitude desfavorável na subcategoria, nas condições de trabalho.

Palavras-chave: atitudes, qualidade de vida no trabalho, saúde ocupacional, bemestar no local de trabalho, a percepção do trabalho, meios de controle do sistema de transporte.

JEL: I31, R41, R42. 


\section{INTRODUCCIÓN}

De acuerdo con el Plan de Ordenamiento Territorial (POT), el sistema de transporte urbano en conjunto con el sistema vial, son un elemento esencial para las ciudades modernas, ya que garantizan la movilidad y el desplazamiento de los usuarios en el entorno urbano. Se ha encontrado a partir de estudios realizados en Latinoamérica, que alrededor del 70\% de los habitantes de las ciudades en desarrollo utilizan el transporte público como su principal medio de movilización (Pardo, 2009: 9), siendo éste uno de los elementos claves dentro de las políticas nacionales, que hace parte de la estructura de las ciudades competitivas y eficientes, y permiten a los ciudadanos estar bajo los principios de economía y desarrollo urbano (Conpes, 2010: 9).

En ese sentido, en el territorio Colombiano, de acuerdo con el Departamento Nacional de Planeación (DNP, 2004: 250) para el año 2019 se busca generar mejores condiciones de vida, salud, educación, empleo, infraestructura, desarrollo, paz y bienestar en las ciudades que conforman el territorio. Dentro de los cambios en infraestructura y desarrollo se ubica al sector transporte a través del proyecto denominado Ciudades amables ${ }^{1}$, con el cual se busca realizar acciones dirigidas al mejoramiento del servicio de transporte público en el país, definiendo para ello, una organización del sistema de acuerdo al número de habitantes de cada ciudad.

De acuerdo al DNP a la ciudad de Pasto le corresponde el funcionamiento del Sistema Estratégico de Transporte Público (SETP). Mediante el Decreto 1079 de 2015 se definen los SETP como los servicios de transporte colectivo integrados y accesibles para la población, los cuales operan de acuerdo a los lineamientos establecidos mediante el Departamento Nacional de Planeación (DNP) y el sistema de Gestión y Control de Flota (SGCF).

El SETP se adoptó en la ciudad mediante el decreto 0562 (Alcaldía de Pasto, 2015: 2), estableciendo dos entes importantes para su ejecución: AVANTE y la Unión Temporal Ciudad Sorpresa (UT). AVANTE es el gestor responsable de la coordinación, e implementación del SETP y la Unión Temporal UT Ciudad Sorpresa como responsable de la operación del SETP de Pasto que coordina la administración de flota y el grupo de operaciones entre los que se encuentran los cargos de supervisor de vía, programador, conductor, control de vía y despachador.

Para el cargo control de vía en los años 2015-2016 se encuentran 94 trabajadores, quienes desempeñan las funciones de controlar las rutas programadas, realizar el seguimiento respectivo en el punto de control que le sea asignado en el área urbana de la ciudad, chequear y revisar la tarjeta de control, establecer con exactitud el tiempo fijado para cada vehículo e informar irregularidades en la ruta.

1 Ciudades amables. proyecto establecido en el año 2005 por el DNP, dentro del documento Visión Colombia II Centenario: 2019 Propuesta para discusión. 
Las personas que desempeñan este cargo son afiliadas al sistema de seguridad y salud en el trabajo SST en el nivel de riesgo IV, que significa alto y cuya gravedad potencial es generar fatalidades y/o lesiones incapacitantes graves. Fasecolda (Federación de Aseguradores Colombianos) en el 2011 brinda un reporte de mortalidad con un número de 11 muertes, y un reporte de accidentes de trabajo con un número de 3.338 en empresas dedicadas al transporte urbano colectivo regular de pasajeros.

Teniendo en cuenta el contexto anterior, es importante referir que anualmente en el mundo se presentan aproximadamente 2.2 millones de muertes asociadas al trabajo, así como lesiones graves en alrededor de 270 millones de personas y 160 millones se enferman por cuestiones laborales (OIT, 2006: 2). Se calcula que los costos económicos por accidentes y enfermedades laborales representan alrededor del 4\% del PIB (Producto Interno Bruto) mundial. Los altos costos en salud en los años recientes han intensificado el interés de los empleadores por fomentar la salud en el trabajo, dado que éste es un aspecto central en la vida de las personas, por lo tanto, éstas deben tener un ambiente seguro y benéfico.

A nivel nacional, a finales del año 2000 mediante el plan de ordenamiento de transporte de la ciudad de Bogotá, se lograron identificar las falencias en el sistema, mencionando que en este sector se presentan cambios y situaciones constantes que llevan a reorganizar el trabajo realizado en la empresa, identificando además, que en algunos casos, se atribuye la responsabilidad al área operativa (despachadores y conductores), quienes han establecido dentro de su cultura organizacional, comportamientos inadecuados respecto a la prestación del servicio y atención al usuario (Burbano; Carvajal, 2005: 323).

En un estudio realizado en Bogotá en el año 2001 frente a las condiciones de trabajo y salud en conductores de una empresa de transporte público urbano, se encontró que las condiciones en el trabajo son deficientes, refiriéndose a la iluminación, el ruido, la exposición a gases y humos, la postura incómoda y el ritmo de trabajo, lo cual según dicho estudio puede propender a los dolores lumbares, enfermedades respiratorias, hipertensión arterial, estrés y ansiedad (Chaparro; Guerrero, 2001: 173). Condiciones que no son alejadas de las personas que desempeñan el cargo control de vía, ya que se encuentran similitudes en cuanto a la exposición de agentes ambientales y las posturas en las cuales desempeñan sus funciones, por lo tanto, el poder visibilizar los riesgos a los cuales se exponen, permite dar línea a otros estudios enfocados en esta población.

De igual manera, en un estudio realizado en Barcelona en el año 2004 se relacionaron factores psicosociales y daños en la salud, donde se reportó que la tensión y el estrés posibilitan una mayor percepción de riesgo en el entorno, lo que puede propiciar accidentes laborales, sumado a la carga y condiciones del trabajo (Salas; Meliá, 2004: 12). Así mismo en un estudio realizado en el sistema de transporte público urbano de México frente a factores psicosociales y patologías laborales en el año 2011, se encontró que los factores de riesgo psicosociales que más perciben los trabajadores en sus funciones son: tareas rutinarias, rapidez de ejecución, servicio al público, horarios extensos de trabajo, condiciones de trabajo desfavorables y estrés (Aranda, et al, 2011: 271). 
En la revisión de investigaciones realizadas a nivel internacional y nacional en el transporte público, se encontró que éstas se han enfocado hacia el cargo de conductores, lo cual evidencia un vacío frente a las categorías de salud laboral, bienestar laboral y percepción del trabajo en otros cargos que hacen parte de este sector, siendo estas características que crean un panorama distinto en cada cargo, puesto que cuentan con elementos específicos que requieren atención, como es el caso del cargo controles de vía. Por lo tanto, el presente artículo busca brindar un panorama inicial sobre las actitudes hacia la calidad de vida laboral en trabajadores que se desempeñan dicho cargo.

La calidad de vida laboral es un concepto multidimensional que abarca diversas condiciones relacionadas con el trabajo, las cuales son relevantes para la satisfacción, la motivación y el rendimiento laboral. Teniendo en cuenta que es un proceso dinámico y continuo, que encamina sus esfuerzos hacia el bienestar de los trabajadores y su entorno, enfatizando en la participación, toma de decisiones, asignación de responsabilidades y garantías laborales (Pazos, 2013: 13). Así, la importancia de trabajar sobre la calidad de vida laboral radica en que esta pretende reconciliar aspectos del trabajo que tienen que ver con las experiencias individuales y con los objetivos organizacionales, además se relaciona con la satisfacción, la salud, el bienestar del trabajador y todo lo concerniente a su entorno laboral (Matabanchoy et al, 2014: 448).

Hoy en día los colaboradores juegan un rol importante en la organización, por lo tanto es necesario innovar en las políticas de gestión del talento humano para responder de manera adecuada y apoyar los objetivos de la empresa potenciando la parte humana (Granados, 2011: 214), con el fin de nutrir el quehacer de la organización en pro del cumplimiento de sus objetivos, preparando a cada individuo para asumir los cambios organizacionales y buscando satisfacer sus necesidades para que logren dar sentido a lo que hacen y se conecten con su trabajo en los niveles emocionales, sociales e intelectuales.

Según Pardo (2009: 11) no existía y en muchos casos no existe una estructura organizacional clara en los sistemas de transporte urbano, lo cual no permite formular estándares de servicio tanto para los usuarios, como garantías para los colaboradores. Igualmente, existen algunos sectores de producción donde es necesario profundizar elementos desde el campo de la psicología del trabajo y las organizaciones, tal es el caso del sector transporte, considerando que existen diversas problemáticas que se pueden abordar a partir de estrategias psicológicas que resulten pertinentes y puedan contribuir al fortalecimiento del sector, partiendo desde una perspectiva de diagnóstico con el fin de evaluar las características que afecten de forma negativa la salud del trabajador.

Finalmente, es importante reconocer que el presente estudio pretende dar una visión inicial frente al cargo control de vía, que permite crear líneas para el fortalecimiento de la calidad de vida laboral, así como aportar un panorama inicial en la empresa, puesto que durante años ha trabajado con ausencia de un área de gestión humana lo cual se ha convertido en una oportunidad de mejora para consolidar diversos procesos enfocados en el bienestar y satisfacción de los colaboradores. De igual manera, busca brindar una perspectiva en la región frente al Sistema de Transporte, dando paso a la visibilización de la 
psicología del trabajo y las organizaciones como un campo que aporta y fortalece diversos escenarios en función del bienestar humano.

\section{MÉTODO}

Se realizó un estudio cuantitativo de carácter descriptivo transversal, el cual consistió en identificar las actitudes de los controles de vía de la Unión Temporal Ciudad Sorpresa de Pasto, hacia los procesos de Calidad de Vida Laboral.

Para recolectar la información se utilizó una escala Likert denominada "Escala de Actitudes hacia la Calidad de Vida Laboral en el SETP de Pasto" (Alfa de Cronbach de 0,86), la cual fue aplicada a 94 controles de vía de la Unión Temporal Ciudad Sorpresa, está compuesta por 108 preguntas que se agrupan en tres categorías, la primera es de salud laboral donde se contemplan condiciones de salud, condiciones de trabajo, estilos de vida y tiempo libre, la segunda es bienestar laboral, y abarca relaciones laborales, familia y clima organizacional, y la última es percepción del trabajo dentro de la cual se encuentran satisfacción laboral y atención al usuario (Oviedo; Sacanambuy; Matabanchoy; Zambrano, comunicación personal, 2015).

La muestra para el presente estudio se realizó por conveniencia, se trabajó con todos los controles de vía pertenecientes a la empresa. El procedimiento en primer lugar consistió en realizar la aplicación de la escala a la totalidad de la población, donde inicialmente se diligenció el consentimiento informado destacando la participación voluntaria en el estudio, se resalta que la investigación se realizó bajo la normatividad de la ley 1090 de 2006 en Colombia, por la cual se menciona la importancia de la confidencialidad en el manejo de la información, que con base en la investigación científica fundamenta sus conocimientos y los aplica en forma válida, ética y responsable en favor de los individuos, los grupos y las organizaciones y al aporte de conocimientos, técnicas y procedimientos para crear condiciones que contribuyan al bienestar y el fortalecimiento de la calidad de vida.

Posteriormente, se realizó la tabulación de los datos y el análisis de la información a partir del programa estadístico IMB SPSS Statics versión 2.0, el cual permitió obtener puntuaciones, agrupaciones y porcentajes de los resultados obtenidos para su posterior análisis y discusión.

\section{RESULTADOS}

Los resultados obtenidos contienen información referente a datos sociodemográficos y morbilidad sentida la cual está compuesta por una ficha de antecedentes médicos y manifestaciones actuales, así como un apartado que hace referencia a las actitudes de los colaboradores que desempeñan el cargo control de vía hacia las categorías de salud laboral, bienestar laboral y percepción del trabajo. 
A continuación se presenta la información recolectada en la ficha de datos sociodemográficos (Ver tabla 1).

Tabla 1. Datos sociodemográficos

\begin{tabular}{|l|l|c|}
\hline \multirow{3}{*}{ Cargo control de vía } \\
\hline \multirow{4}{*}{ Sexo } & Mujer & $71,1 \%$ \\
\cline { 2 - 3 } & Hombre & $25,5 \%$ \\
\hline \multirow{4}{*}{ Ediempo vinculado a la empresa } & 1 a 5 años & $38,8 \%$ \\
\cline { 2 - 3 } & 1 año o menos & $18,8 \%$ \\
\hline \multirow{5}{*}{ Escolaridad } & 50 a 60 años & $12,8 \%$ \\
\cline { 2 - 3 } & 40 a 50 años & $21,3 \%$ \\
\cline { 2 - 3 } & 30 a 40 años & $35,1 \%$ \\
\cline { 2 - 3 } & 20 a 30 años & $25,5 \%$ \\
\hline \multirow{4}{*}{ Estado civil } & Secundaria & $84 \%$ \\
\cline { 2 - 3 } & Técnico & $17,7 \%$ \\
\cline { 2 - 3 } & Tecnológico & $2,1 \%$ \\
\hline \multirow{5}{*}{ Hijos } & Soltero & $44 \%$ \\
\cline { 2 - 3 } & Casado & $36 \%$ \\
\cline { 2 - 3 } & Unión libre & $20 \%$ \\
\hline
\end{tabular}

A continuación se presenta la información recolectada en el autoreporte de morbilidad sentida (Ver tabla 2), que aporta a la categoría de salud laboral, y se compone de antecedentes médicos y manifestaciones actuales.

Tabla 2. Autoreporte de morbilidad sentida

\begin{tabular}{|l|c|}
\hline \multicolumn{2}{|c|}{ Autoreporte de morbilidad sentida } \\
\hline Dolores de espalda & $48,9 \%$ \\
\hline Dolores de rodilla & $48,9 \%$ \\
\hline Dolores de cabeza & $45,7 \%$ \\
\hline Cansancio visual & $47,9 \%$ \\
\hline Dolores de cuello & $42,6 \%$ \\
\hline Cansancio o aburrimiento & $40 \%$ \\
\hline
\end{tabular}


En el apartado de morbilidad sentida (antecedentes médicos y manifestaciones actuales), los dolores de espalda son los más frecuentes $(48,9 \%)$, seguido de dolores de rodilla $(48,9 \%)$, dolores de cabeza $(45,7 \%)$, cansancio visual $(47,9 \%)$ y dolores de cuello $(42,6 \%)$ siendo estos las molestias más frecuentes en quienes desempeñan el cargo control de vía. En cuanto a manifestaciones actuales, la variable con el porcentaje más elevado fue cansancio o aburrimiento con el $40 \%$.

A continuación se presenta la información encontrada en la categoría de salud laboral (Ver tabla 3), que se compone de las subcategorías de condiciones de salud, condiciones de trabajo, estilos de vida y tiempo libre.

\section{Tabla 3. Salud Laboral}

Salud Laboral

Condiciones de Salud

\begin{tabular}{|c|c|c|}
\hline Actitud & Frecuencia & Porcentaje \\
\hline Muy favorable & 60 & $63,8 \%$ \\
\hline Favorable & 15 & $15,9 \%$ \\
\hline Ni favorable, ni desfavorable & 16 & $17 \%$ \\
\hline Desfavorable & 1 & $1 \%$ \\
\hline Muy desfavorable & 2 & $2,1 \%$ \\
\hline \multicolumn{3}{|l|}{ Condiciones de Trabajo } \\
\hline Actitud & Frecuencia & Porcentaje \\
\hline Muy favorable & 5 & $5,3 \%$ \\
\hline Favorable & 12 & $12,7 \%$ \\
\hline Ni favorable, ni desfavorable & 12 & $12,7 \%$ \\
\hline Muy desfavorable & 65 & $69,1 \%$ \\
\hline \multicolumn{3}{|l|}{ Estilos de Vida } \\
\hline Actitud & Frecuencia & Porcentaje \\
\hline Muy favorable & 27 & $28,7 \%$ \\
\hline Favorable & 15 & $15,9 \%$ \\
\hline Ni favorable, ni desfavorable & 30 & $31,9 \%$ \\
\hline Desfavorable & 9 & $9,5 \%$ \\
\hline Muy desfavorable & 10 & $10,6 \%$ \\
\hline Tiempo libre & & \\
\hline
\end{tabular}




\begin{tabular}{lcc}
\hline \multicolumn{1}{c}{ Actitud } & Frecuencia & Porcentaje \\
\hline Muy favorable & 26 & $27,6 \%$ \\
\hline Favorable & 14 & $14,8 \%$ \\
\hline Ni favorable, ni desfavorable & 37 & $39,3 \%$ \\
\hline Desfavorable & 10 & $10,6 \%$ \\
\hline Muy desfavorable & 5 & $5,3 \%$ \\
\hline
\end{tabular}

Los resultados sobre las condiciones de salud muestran que el 63,8\% de los colaboradores presentan una actitud muy favorable con respecto a recibir información de factores que intervienen sobre la salud; entre los factores más importantes para ellos están los beneficios de realizar actividad física (81\%), prevención de enfermedades de transmisión sexual (78\%), prevención de la hipertensión arterial (77\%), higiene oral (77\%), consumo de alcohol (74\%), consumo de cigarrillo (74\%), prevención de la diabetes (74\%), consumo de sustancias psicoactivas (73\%), y prevención de la obesidad (73\%), siendo estos resultados un factor protector dentro de la subcategoría.

En cuanto a condiciones de trabajo el 69,1\% de los controles de vía muestran una actitud muy desfavorable hacia las condiciones de trabajo, dentro de esta subcategoría considera importante que se realice una inspección de riesgos en el lugar en que laboran $(81,9 \%)$, se mejoren las condiciones físicas del lugar donde trabajan $(77,2 \%)$, además perciben que se encuentran expuestos a frío dentro de su lugar de trabajo $(74,2 \%)$, y consideran importante conocer las normas de seguridad para evitar las enfermedades causadas por el trabajo (87\%), así como las normas de tránsito para evitar accidentes en el vehículo (86,2\%), esta información permite identificar los factores que inciden en su salud, posibilitando en un futuro la planeación de estrategias para minimizar sus efectos.

Respecto a los estilos de vida, el 28,7\% de la población muestra una actitud muy favorable en cuanto a los hábitos o comportamientos que determinan el estado de salud. El 98,9\% no consumen ningún tipo de sustancias psicoactivas, el 80\% consideran importante que en la empresa se promueva actividades recreativas, el 80,9\% consideran importante practicar ejercicio físico y el 79,1\% de las personas que desempeñan el cargo control de vía consumen alimentos preparados en casa.

En la categoría de tiempo libre el 39,3\% de los controles expresan tener una actitud indiferente frente a las actividades que realizan fuera de su trabajo, dentro de estas actividades, las que se realizan con mayor frecuencia son, dormir $(57,4 \%)$, compartir con sus amigos $(53,8 \%)$, ver películas $(51,1 \%)$, salir a bailar $(47,9 \%)$ y salir de paseo $(47,8 \%)$.

A continuación se presenta la consolidación de la información respecto a la categoría de bienestar laboral (Ver tabla 4) que se compone de las subcategorías de relaciones laborales, familia y clima organizacional. 
Tabla 4. Bienestar laboral

\begin{tabular}{|c|c|c|}
\hline \multicolumn{3}{|c|}{ Bienestar laboral } \\
\hline \multicolumn{3}{|c|}{ Relaciones laborales } \\
\hline Actitud & Frecuencia & Porcentaje \\
\hline Muy favorable & 12 & $12,7 \%$ \\
\hline Favorable & 10 & $10,6 \%$ \\
\hline Ni favorable, ni desfavorable & 47 & $50 \%$ \\
\hline Desfavorable & 10 & $10,6 \%$ \\
\hline Muy desfavorable & 12 & $12,7 \%$ \\
\hline \multicolumn{3}{|l|}{ Familia } \\
\hline Actitud & Frecuencia & Porcentaje \\
\hline Muy favorable & 52 & $55,3 \%$ \\
\hline Favorable & 27 & $28,7 \%$ \\
\hline Ni favorable, ni desfavorable & 13 & $13,8 \%$ \\
\hline Desfavorable & 2 & $2,1 \%$ \\
\hline \multicolumn{3}{|l|}{ Clima organizacional } \\
\hline Actitud & Frecuencia & Porcentaje \\
\hline Muy favorable & 54 & $57,4 \%$ \\
\hline Favorable & 15 & $15,9 \%$ \\
\hline Ni favorable, ni desfavorable & 13 & $13,8 \%$ \\
\hline Desfavorable & 2 & $2,1 \%$ \\
\hline Muy desfavorable & 10 & $10,6 \%$ \\
\hline
\end{tabular}

El $50 \%$ de los controles de vía afirman tener una actitud indiferente frente a las relaciones laborales. También consideran sentirse orgullosos de su oficio $(85,1 \%)$, así como de pertenecer al Sistema Estratégico de Transporte Público de Pasto (88,3\%).

El 55,3\% de los colaboradores presentan una actitud muy favorable en la subcategoría familia, manifiestan sentirse a gusto conviviendo con ella $(90,4 \%)$, también perciben que sus familias tienen confianza en ellos (76,6\%), sienten apoyo emocional ante cualquier situación (76,3\%), y consideran que sus familias son comprensivas (72\%).

En cuanto a clima organizacional el 57,4\% de los controles de vía manifiestan una actitud muy favorable frente a las percepciones que tienen respecto a la empresa. Consideran que sus jefes de trabajo son respetuosos $(86,2 \%)$, y que es importante poner en práctica valores como: la honestidad $(93,5 \%)$ y el respeto $(88,3 \%)$, así como orientar a las personas para que se sientan bien en su puesto de trabajo $(90,4 \%)$, realizar capacitaciones para mejorar la comunicación efectiva $(86,2 \%)$ y realizar capacitaciones para mejorar la resolución de conflictos (86\%). 
A continuación se presentan los resultados de la categoría percepción del trabajo (Ver tabla 5), que se compone de las subcategorías de satisfacción laboral y de atención al usuario.

Tabla 5. Percepción del trabajo

\begin{tabular}{|c|c|c|}
\hline \multicolumn{3}{|c|}{ Percepción del trabajo } \\
\hline \multicolumn{3}{|c|}{ Satisfacción laboral } \\
\hline Actitud & Frecuencia & Porcentaje \\
\hline Muy favorable & 14 & $14,8 \%$ \\
\hline Favorable & 20 & $21,2 \%$ \\
\hline Ni favorable, ni desfavorable & 38 & $40,4 \%$ \\
\hline Desfavorable & 5 & $5,3 \%$ \\
\hline Muy desfavorable & 17 & $18,0 \%$ \\
\hline \multicolumn{3}{|l|}{ Atención al usuario } \\
\hline Actitud & Frecuencia & Porcentaje \\
\hline Muy favorable & 88 & $93,6 \%$ \\
\hline Favorable & 3 & $3,1 \%$ \\
\hline Ni favorable, ni desfavorable & 3 & $3,1 \%$ \\
\hline
\end{tabular}

El 40,4\% de los controles de vía tienen una actitud de indiferencia hacia la subcategoría de satisfacción laboral. Consideran que las funciones que cumplen les gustan (84\%), que el trabajo ocupa una parte importante en sus vidas $(81,7 \%)$ y que se sienten motivados por éste $(76,6 \%)$.

En atención al usuario el 93,6\% de los controles de vía, tienen una actitud muy favorable frente a capacitarse en este tema, considerando que, una mejor atención ayuda a atraer más usuarios (93,6\%), también se encontró que el saludo ayuda a que las personas se sientan más a gusto con el servicio $(93,6 \%)$, y el buen trato los lleva a preferir el servicio del bus colectivo $(92,5 \%)$.

\section{CONCLUSIONES}

Los resultados encontrados permiten visibilizar y describir las actitudes hacia la calidad de vida laboral en 94 trabajadores que desempeñan el cargo control de vía en la Unión Temporal Ciudad Sorpresa, teniendo como base las categorías de salud laboral, bienestar laboral y percepción laboral.

Se entiende la Calidad de vida laboral como el proceso de humanizar el lugar de trabajo y la satisfacción de las necesidades de los colaboradores (Matabanchoy et al, 2014: 448). Este concepto se relaciona con la satisfacción, la salud, el bienestar y todo lo concerniente al 
entorno de trabajo. En el presente estudio se encontró que el 57,1\% de la población muestra una actitud muy favorable hacia la calidad de vida laboral, lo cual permite reconocer que la organización está asumiendo los compromisos dirigidos a satisfacer las necesidades de sus trabajadores, sin embargo, existen diversos factores que requieren atención, pues suponen un reto para la misma, esto con el fin de lograr que las personas se conecten con su trabajo en los niveles emocionales, sociales e intelectuales, e influya de manera positiva en el bienestar de los colaboradores y por ende en la productividad de la organización.

En cuanto a antecedentes médicos se encontró que las molestias más frecuentes en las personas que desempeñan el cargo control de vía son dolores de espalda, rodilla, cabeza, cuello y cansancio visual, factores que inciden en el bienestar del colaborador. Luttmann (2014: 8) los define como trastornos musculoesqueléticos, aquellos problemas de salud que afectan el aparato locomotor, lo cual abarca todo tipo de dolencias, desde las molestias leves y pasajeras hasta las lesiones irreversibles y discapacitantes, lo anterior permite analizar la importancia de desarrollar en la organización ambientes laborales saludables, enfocados en la promoción de la salud y prevención de enfermedades.

De acuerdo con el Ministerio de Trabajo, se entiende las condiciones laborales como aquellos factores identificables de situaciones adversas de seguridad y salud en el lugar de trabajo, siendo necesario realizar una evaluación del puesto, buscando mantener el bienestar de los trabajadores y previniendo el daño causado a la salud (OIT, 2006: 6), en este sentido, los controles de vía, muestran una actitud muy desfavorable, refiriendo, que se debe realizar un análisis de los puestos de trabajo (81,9\%), así como una mejora en las condiciones físicas del lugar donde trabajan $(77,2 \%)$. Lo anterior invita a reflexionar sobre la responsabilidad que tiene la organización en la realización de acciones encaminadas a la mejora de las condiciones en el lugar de trabajo ya que estas inciden directamente en la salud del trabajador, según la OMS (2007: 6) la protección de la salud en el lugar de trabajo requiere la promulgación de reglamentos y la adopción de un conjunto básico de normas de salud ocupacional a fin de asegurar que en todos los lugares de trabajo se cumplan los requisitos mínimos de protección, para esto se deben tener en cuenta las necesidades específicas del cargo.

Los estilos de vida son aquellos hábitos y comportamientos sostenidos de individuos y grupos sociales (Álvarez, et al, 2006: 4), los cuales son determinados, aprendidos y adoptados a través de un proceso de aprendizaje y socialización, encontrándose que es el contexto y los recursos disponibles en el medio los que determinan el comportamiento (Meza, et al, 2012: 53). Se encontró que las actitudes de los controles de vía se ubicaron en "ni favorable, ni desfavorable" (31,9\%), lo cual podría indicar actitudes de indiferencia, al igual que la categoría de tiempo libre con una puntuación 39,3\%, los anteriores elementos pueden estar relacionados, pues entre los factores de riesgo de los estilos de vida saludables se encuentran: la vida sedentaria, el tabaquismo, alcoholismo y el uso del tiempo libre, lo anterior, indica una posible falta de compromiso tanto a nivel individual como social, ya que el promover y adquirir estilos de vida saludables es un actividad conjunta que contribuye a la mejora en la calidad de vida. Sin embargo se recomienda emplear otras estrategias que permitan conocer a detalle los factores que influyen y que a su vez determinan las actitudes de los colaboradores hacia estas categorías. 
En el presente estudio el 50\% de la población afirma tener una actitud de indiferencia frente a las relaciones laborales con sus compañeros y la gerencia de la organización. Por su parte Trebilcock (1998: 21) menciona que las relaciones laborales son el sistema en el que las empresas, los trabajadores y sus representantes, directa o indirectamente, interactúan con el fin de establecer las normas básicas que rigen las relaciones de trabajo, siendo estas un eje fundamental para el adecuado desarrollo organizacional. Lo anterior, invita a la organización a empezar a trabajar en relaciones laborales, éstas, dirigidas hacia un óptimo clima organizacional teniendo en cuenta que este influye en otros aspectos que involucran a los trabajadores y a la organización. La responsabilidad para este fin es bidireccional, corresponde tanto a los empleados como a la gerencia, teniendo en cuenta reglas básicas de comunicación y la disposición que se tenga frente al cambio, para que así, en un futuro el trabajador logre percibir apoyo de sus compañeros de trabajo y la gerencia.

Muñoz, (2014: 23) define el sentido de pertenencia como la aptitud de considerarse y de sentirse integrante de un grupo, en la organización, se puede identificar como la relación empresa - trabajador. Esta relación se orienta hacia la realización de los objetivos individuales y organizacionales. Cuando estos dos objetivos convergen, se satisfacen mutuamente y existe responsabilidad social por parte de la organización y produce bienestar en los trabajadores, generando así sentido de pertenencia a la empresa. En el presente estudio se encontró que los controles de vía tienden a sentirse orgullosos de su oficio con un 85,1\%, así como al pertenecer al Sistema Estratégico de Transporte Público de Pasto con un 88,3\%. Esto muestra que las personas que desempeñan el cargo, se sienten en gran medida identificados con la organización, siendo este un eje clave para comenzar a potencializar otros factores que contribuyen a su calidad de vida laboral.

El 55,3\% de la población demuestra tener una actitud muy favorable en cuanto a la subcategoría familia. Según Idrovo (2006: 52) las empresas que adoptan programas de apoyo familiar permiten crear una fuerza de trabajo comprometida, esfuerzo que indudablemente facilitará la retención de profesionales. Las diversas investigaciones y estudios demuestran que en general, la implementación de políticas de conciliación trabajo - familia, está asociada con resultados positivos. Partiendo desde esta perspectiva la familia es un factor protector para las personas que desempeñan el cargo control de vía, ya que disminuye la probabilidad de riesgo y genera bienestar, por ende, la organización debe iniciar con la implementación de programas donde se la vincule para que el trabajador sienta que ésta se interesa por él y su núcleo primario.

De acuerdo con Chiang, et al, (2008), el clima laboral se refiere a las percepciones en cuanto a políticas, prácticas y procedimientos organizacionales. En este sentido el 57,4\% de los controles de vía manifiestan una actitud muy favorable frente a las percepciones que tienen respecto a la empresa, según Páez (2014: 8) un clima organizacional adecuado hace que la empresa sea permeable a cualquier cambio que presente en todos sus procesos, donde cada uno de los proyectos que se desarrollan en la organización se vuelven retos personales y de alta cooperación entre los colaboradores, lo cual sugiere que trabajar por un buen clima organizacional es muy rentable y beneficioso para cualquier organización. 
En lo relacionado con la percepción del trabajo se encontró que los niveles de satisfacción laboral son altos con un $89,4 \%$, lo que lleva a considerar que las personas sienten que las funciones que desempeñan, las expectativas frente al rol en la organización y las posibilidades de progreso son propicias para su desarrollo personal, además muestran actitudes positivas ante las tareas y la organización. Según Atalaya (1999) lo anterior indica que hay un aumento en el compromiso del trabajador puesto que la satisfacción laboral comprende diversos componentes tales como las condiciones de trabajo, el esfuerzo empleado en la tarea, los salarios y la posibilidad del desarrollo personal, la cual a su vez afecta actitudes ante la vida, la familia y sí mismo.

Los controles de vía por su parte afirman que las funciones que cumplen les gustan con un $84 \%$. Para el $81,7 \%$ el trabajo ocupa una parte importante de sus vidas y el 76,6\% consideran que se sienten motivados por las tareas que desempeñan. Por lo tanto, el apoyo recibido de la empresa es indispensable, debido a que permite fortalecer el ambiente de trabajo, la relación con sus compañeros y la motivación, además permite reducir los efectos de algunos estresores laborales, aumentar el bienestar de los colaboradores, y a su vez, mejorar otros aspectos, como, la satisfacción laboral y el compromiso con el trabajo (López; Moreno, 2013: 118).

El 53\% de los controles de vía manifestaron que el trabajo en algunas ocasiones es desgastante, lo cual aumenta los niveles de estrés de manera considerable, por tanto es importante llegar a un equilibrio entre estos factores para que logren ser vistos como un reto y no creen frustración o situaciones de fracaso que afecten la satisfacción y la motivación (Fuentes, 2012: 22). Se considera fundamental identificar la relación entre calidad de vida laboral y satisfacción laboral, puesto que la organización se reconoce como un sistema abierto y dinámico, donde el éxito depende en gran medida de lo que las personas hacen y cómo lo hacen, pues busca potenciar el trabajo y los factores que lo rodean promoviendo un desarrollo integral, de manera individual y grupal, para conseguir el crecimiento de la organización y el bienestar laboral.

El 93,6\% de los controles de vía muestran una actitud muy favorable hacia recibir capacitaciones en atención al usuario, ésta es indispensable pues tal como lo afirma la fundación CETMO (2006: 1), para que el transporte progrese de manera significativa, debe estar totalmente orientado al servicio y por ende responder ante las necesidades de sus usuarios, con lo anterior se identifica la apertura que los controles de vía tienen por mejorar la prestación del servicio, lo cual es un llamado a la organización para aprovechar y estimular dicha motivación en pro de la optimización del servicio.

Los resultados obtenidos permiten identificar las necesidades de la población estudiada con el fin de crear líneas de intervención dirigidas a mejorar la calidad de vida laboral, teniendo en cuenta que la salud laboral, el bienestar laboral y la percepción del trabajo benefician a las personas que desempeñan el cargo y a su vez a la organización.

El presente trabajo permitió la identificación, descripción y análisis de las actitudes de los controles de vía hacia la Calidad de Vida Laboral, brindando un panorama inicial en la 
región, así como insumos a la organización sobre los cuales trabajar con el fin de mejorar el Sistema de Transporte Urbano.

Durante el proceso se identificó la necesidad de potenciar el área de talento humano ya que contribuye y brinda herramientas que fortalecen de manera significativa la Calidad de Vida Laboral.

Para futuras investigaciones se recomienda abordar todas las áreas de la organización con el fin de obtener un diagnóstico completo que permita visibilizar las necesidades y fortalezas en función de la mejora en la calidad de vida laboral.

\section{REFERENCIAS}

(1) ALCALDÍA DE PASTO. (2015). Decreto 0562 del 26 de agosto de 201. En: Alcaldía de Pasto, pp. 2-23. [En línea] Disponible en http://www.pasto.gov.co/index.php/transito-y-transporte/6103-decreto-0562-adopta-sistema-estrategico-de-transporte-publico (consultado may. 2-06).

(2) ÁLVAREZ, Irene (2006). "Estilos de vida saludable y no saludable que tienen los y las trabajadoras del núcleo de procesos artesanales del instituto nacional de aprendizaje. Enfermería Actual en Costa Rica”. En: Revista en enfermería actual. Año/vol. 5, No. 10. San José, Costa Rica. Pp. 1-21.

(3) ARANDA, Carolina; PANDO, Manuel; TORRES, Teresa; SALAZAR, José; SÁNCHEZ, José. (2011)." Factores psicosociales y patologías laborales en trabajadores de un sistema de transporte público urbano en México". En: Revista Colombiana de Psiquiatría. Año/vol. 40, No. 002. Bogotá, Colombia. Pp. 266-278.

(4) ATALAYA, María (1999). “Satisfacción laboral y productividad”. En: Revista de Psicología Año/vol. 3, No. 5. Perú. Pp. 6-46.

(5) BURBANO, Johanna; CARVAJAL, Luz. (2005). "Empresas de transporte: entre transmilenio y la incertidumbre”. En: Pontificia Universidad Javeriana. Octubre-Diciembre, año/vol. 4, No. 003. Bogotá, Colombia. Pp. 317-324.

(6) CETMO (2006). "Recomendaciones para la mejora de la satisfacción del cliente de transporte público" En: Buenas prácticas y recomendaciones para la mejora de la satisfacción del cliente de transporte público de viajeros por carretera. Noviembre, pp. 1-28. [En línea] Disponible en http://www.fundacioncetmo.org/ dgt\%20 calidad\%20viajeros/pdf/buenas.practicas/cap.3.Recomendaciones.pdf (consultado ago.10-06).

(7) CHAPARRO, Pablo; GUERRERO, Juan. (2001). “Condiciones de Trabajo y Salud en Conductores de una Empresa de Transporte Público Urbano”. En: Revista de Salud Pública. Año/vol. 3. Bogotá, Colombia. Pp. 171187. 
(8) CHIANG, María; SALAZAR, Mauricio; HUERTA, Patricia; NUÑEZ, Antinio. (2008). “Clima organizacional y satisfacción laboral en organizaciones del sector estatal (Instituciones Públicas) Desarrollo, adaptación y validación de instrumentos". En Revista Universum. Año/vol. 2, No. 23. Chile. Pp. 66-85.

(9) CONPES (Consejo Nacional de Política Económica y Social). (2010). Sistema Estratégico de Transporte Público de pasajeros para el municipio de Valledupar. En: Ministerio de Hacienda y Crédito Público. Ministerio de Transporte, pp. 2-422. [En línea] Disponible en http://datateca.unad.edu.co/contenidos/102902/ CONPES_3656.pdf (consultado jun. 13-06).

(10) DNP (Departamento Nacional de Planeación) (2004). Construir ciudades amables. En Visión Colombia Segundo Centenario, pp. 247-262. [En línea] Disponible en http://www.mineducacion.gov.co/cvn/1665/ articles-95980_archivo_pdf17.pdf (consultado may. 2-06).

(11) FASECOLDA. (2011). Reporte de mortalidad. En: Federación de aseguradores Colombianos. [En línea] Disponible en el sitio web de la Federación de Aseguradores Colombianos en http://www.fasecolda.com/index.php/ramos/automoviles/informe-de-reclamaciones/ (consultado may. 2-06).

(12) FUENTES, Silvia (2012). “Satisfacción laboral y su influencia en la productividad”. En: Universidad Rafael Landívar. Marzo, pp. 1-97. [En línea] Disponible en http://biblio3.url.edu.gt/Tesis/2012/05/43/Fuentes-Silvia.pdf (consultado sep. 13-06).

(13) GRANADOS, Isabel. (2011). “Calidad de Vida Laboral: historia, dimensiones y beneficios”. En: Revista IIPSI. Año/vol. 14, No. 002. Lima, Perú. Pp. 214-225.

(14) IDROVO, Sandra (2006). "Las políticas de conciliación trabajo-familia en las empresas Colombianas". En: Estudios gerenciales. Julio-Septiembre, año/vol. 22, No. 100. Pp. 50-70.

(15) LEY 1090 (2006). “De la profesión de Psicología”. [En línea] Disponible en http://www.psicologiaprospectiva.com/introley1090.html (consultado en jul. 27-06).

(16) LÓPEZ, Cinthya; MORENO, Marjorie (2013). "Instrumento para evaluar variables psicológicas y laborales en conductores de autobuses”. En: Reflexiones, año/vol. 92, No. 2. Costa Rica. Pp. 109-122.

(17) LUTTMAN, Alwin (2001). “Prevención de trastornos musculoesqueleticos en el lugar de trabajo”. En: Federal Institute for Occupational Safety and Healt. Núm. 5, pp. 1-32. [En línea] Disponible en: http://www.who. int/occupational_health/publications/en/pwh5sp.pdf (consultado sep. 14-06).

(18) MATABANCHOY, Sonia; CALDERÓN, Gregorio; BETANCOURT, Sonia. (2014). Aproximaciones a la reflexión sobre la calidad de vida laboral. En: Psicología de las organizaciones y del trabajo. Editorial Bonaventuriana. Valle del Cauca.

(19) MEZA, Mary; VILLALOBOS, Grettel; NÚÑEZ, Natalia; OROZCO, Uvania; QUIEL, Sharon; ZÚÑIGA, Gabriela. (2012). "Análisis sobre estilos de vida, ambiente laboral, factores protectores y de riesgo en seis grupos poblacionales de una empresa transnacional en el periodo 2005-2008”. En: Enfermería en Costa Rica, año/ vol. 32, No. 002. Costa Rica. Pp. 51-58.

(20) MUÑOZ, Paola (2014). "El sentido de pertenencia como estrategia organizacional para el aporte de los objetivos de la empresa”. En: Universidad Militar Nueva Granada, pp. 1-30. [En línea] Disponible en http:// repository.unimilitar.edu.co/bitstream/10654/11663/1/Ensayo\%20Final\%20Paola\%20Mu\%C3\%B1oz. pdf (consultado sep. 23-06).

(21) O.I.T. Organización Internacional del Trabajo. (2006). Consejo de administración para debate y orientación. Seguridad y salud en el trabajo: sinergia entre la seguridad y la productividad. En: Oficina Internacional del Trabajo, pp. 2-15. [En línea] Disponible en http://www.bvsde.paho.org/bvsacd/cd65/seguridad.pdf (consultado may. 7-06).

(22) OMS Organización Mundial de la Salud (2007). "Salud de los trabajadores: plan de acción mundial”. En: 60 Asamblea Mundial de la Salud, pp. 1-9. [En línea] Disponible en http://www.who.int/occupational_health/ WHO_health_assembly_sp_web.pdf (consultado sep. 29-06). 
(23) OVIEDO, Viviana; SACANAMBUY, Jose; MATABANCHOY, Sonia; ZAMBRANO, Christian. (2015). Escala de Evaluación de la Calidad de Vida en el Sistema Integrado de Transporte de Pasto. Pasto, Nariño. Comunicación no oficial.

(24) PAEZ, Johana (2014). "El clima organizacional y su influencia en los proyectos de alto impacto de una organización". En: Universidad Militar Nueva Granada, Noviembre, pp. 1-20. [En línea] Disponible en http:// repository.unimilitar.edu.co/bitstream/10654/13142/1/ARTICULO\%20FINAL\%206501671..pdf (consultado sep. 28-06).

(25) PARDO, Carlos. (2009). Los cambios en los Sistemas Integrados de Transporte Masivo en las principales Ciudades de América Latina. Publicaciones de las Naciones Unidas. En: CEPAL Colección Documentos de Proyectos. [En línea] Disponible en http://repositorio.cepal.org/bitstream/handle/11362/3641/ S2009308_es.pdf?sequence=1 (consultado jun. 13-06).

(26) PAZOS, Ana. (2013). Calidad de Vida Laboral de un grupo de personas en situación de discapacidad. En: Universidad del Valle, pp. 1-93. [En línea] Disponible en http://bibliotecadigital.univalle.edu.co/bitstream/10893/8757/1/CALIDAD\%20DE\%20VIDA\%20LABORAL-UNIVALLE.pdf (consultado ago. 10-06)

(27) SALAS, Carles; MELIÁ, Josep. (2004). Evaluación de riesgos psicosociales y factores psicosociales que afectan la probabilidad de accidente en una empresa de Transporte Urbano de Economía Social. En: Tercer congreso internacional de riesgos laborales, pp. 1-15. [En línea] Disponible en http://www.uv.es/meliajl/ Papers/2004SalasMelia.pdf (consultado abr. 14-06).

(28) TREBILCOCK, Anne (1998). “Relaciones laborales y gestión de recursos humanos: visión general”. En: Enciclopedia de Salud y Seguridad en el Trabajo, pp. 21.2-21.9. [En línea] Disponible en http://www.insht.es/ InshtWeb/Contenidos/Documentacion/TextosOnline/EnciclopediaOIT/tomo1/21.pdf (consultado sep. 20-06). 